\title{
Parameter Estimation Algorithm for the Exponential Signal by the Enhanced DFT Approach
}

\author{
Qian Wang ${ }^{1}$, Xiao Yan $^{2}$, Kaiyu Qin ${ }^{1}$ \\ ${ }^{1}$ School of aeronautics and astronautics, University of Electronic Science and Technology of China, Xiyuan Ave, West Hi- \\ Tech Zone, No.2006, 611731, Chengdu, China, job_wangqian@uestc.edu.cn \\ ${ }^{2}$ School of aeronautics and astronautics, University of Electronic Science and Technology of China, Xiyuan Ave, West Hi- \\ Tech Zone, No.2006, 611731, Chengdu, China, yanxiao@uestc.edu.cn
}

\begin{abstract}
Based on enhanced interpolation DFT, a novel parameter estimation algorithm for the exponential signal is presented. The proposed two-step solution consists of a preprocessing unit which constructs a new signal sequence by continuously cycle shifting sample points and summing up $N$ buffered exponential signal sample sequences, then an interpolation DFT engine to obtain accurate parameter estimation of the exponential signal based on the signal sequence generated by the preprocessing unit. Compared to previous works, owing to the combination of the pretreatment and the interpolation DFT analysis, the tedious iteration for spectral leakage elimination can be removed, and remarkable improvements are achieved in terms of estimation accuracy and speed. The simulation results verify the effectiveness of the proposed algorithm.
\end{abstract}

Keywords: Parameters estimation, exponential signal, cycle shift, discrete Fourier transform (DFT).

\section{INTRODUCTION}

$\mathrm{S}$ INCE THE exponential signal model has been widely used in many engineering applications, such as linear system identification, electric power system analysis [1], radar and sonar systems, cognitive radio, nuclear magnetic resonance spectroscopy (NMRS) [2], etc., its characteristic parameters, including frequency, amplitude, damping and phase, are often required to be analyzed and estimated accurately [3]. The general estimation procedures for these parameters can be classified as either parametric or nonparametric methods. Parametric methods, such as autoregressive models, Prony's method, MUSIC algorithm, etc., can provide high-accuracy estimates, however, they demand intensive computational algorithms to realize prefect 'model agreement' with a real multi-component signal [4]. Nonparametric methods are computationally efficient and have less sensitivity to algorithm design parameters. Applying the discrete Fourier transform (DFT) to estimation of damped exponential signal is the primary approach of the nonparametric estimation.

In order to achieve the high-accuracy estimates using a DFT-based method, the inherent limitations of DFT due to the picket fence and spectral leakage effects caused by noncoherent sampling, which may result in unacceptable estimation errors, must be worked out. To counteract the picket fence effect of DFT, the interpolated DFT (IpDFT) method which can mitigate the impact of sampling of the continuous spectrum is introduced [5], [6]. The spectrum samples are suitably interpolated in the neighborhood of each spectral peak. Although the picket fence effect suppression is improved with the increase of interpolation points in [7], [8], the influence of spectral leakage cannot be alleviated simultaneously. Then, based on three maximum DFT bin interpolation, the spectral leakage is reduced by means of iterative estimation and subtraction of the negative part of the spectrum [9]. However, the iteration is extremely intensive computationally and difficult to implement. On the other hand, since the window sequence is usually employed before DFT to suppress the spectral leakage effect, the different time windows are investigated for the parameter estimation [10]-[13]. Unfortunately, a particular window cannot improve the estimation accuracy of all parameters simultaneously, some compromise must be made. The rectangular window for its high frequency resolution is widely used for frequency estimation [7]-[9]. A weighted multipoint interpolation of the DFT algorithm with the Hanning window is proposed by [10], and it can only improve the accuracy of the amplitude estimation. Recently, a new DFT interpolation algorithm, the so-called RVCI-M (Rife-Vincent algorithm) for signal analysis, was derived [11]. RVCI-M which utilizes the Rife-Vincent class-I window has a preferable frequency estimate, but is not good at precise damping factor estimation.

In this paper, a new parameter estimation algorithm for the exponential signal, which is based on the enhanced DFT approach, is presented. The proposed algorithm first constructs a new signal sequence by continuously cycle shifting sample points and summing up $N$ buffered exponential signal sample sequences. Then, according to the new signal sequence, the interpolated DFT refers to the three neighboring spectral lines at the peak of each mode to obtain accurate parameter estimation of the exponential signal. Compared to previous works, owing to the combination of the pretreatment and the interpolation DFT analysis, the tedious iteration for spectral leakage elimination can be removed, and remarkable improvements are achieved in terms of estimation accuracy. The performance of the proposed algorithm is validated by simulation, and the comparison of different algorithms is also discussed in detail.

The following sections are organized as follows. In Section 2, the proposed parameter estimation algorithm is presented and discussed in detail. Performance assessments and comparison under different conditions are provided in Section 3. Finally, the paper is concluded in the last section. 


\section{THE PROPOSED ALGORITHM}

\section{A. Signal preprocessing.}

The discrete signal $x(n)$ is transformed into the frequency domain by DFT, which can be defined as

$$
X(m)=\sum_{n=0}^{N-1} x(n) e^{-j 2 \pi m n / N}, m=0,1, . ., N-1
$$

where $N$ is the number of samples.

For a given sample $x(N-1)$ in the discrete exponential signal sequence, there are $N N$-dimensional vectors containing this sample element.

$$
\begin{gathered}
\boldsymbol{x}_{0}=[x(N-1), x(N), \cdots, x(2 N-2)] \\
\boldsymbol{x}_{1}=[x(N-2), x(N-1), \cdots, x(2 N-3)] \\
\boldsymbol{x}_{N-1}=[x(0), x(1), \cdots, x(N-1)]
\end{gathered}
$$

The elements of each vector are then cycle shifted to guarantee that the given sample $x(N-1)$ is the first element of each vector. The cycle shifted vectors can be expressed as:

$$
\begin{gathered}
\boldsymbol{x}_{0}^{(0)}=[x(N-1), x(N), \cdots, x(2 N-2)] \\
\boldsymbol{x}_{1}^{(1)}=[x(N-1), \cdots, x(2 N-3), x(N-2)] \\
\boldsymbol{x}_{N-1}^{(N-1)}=[x(N-1), x(0), \cdots, x(N-2)]
\end{gathered}
$$

where $\boldsymbol{x}_{n}^{(n)}$ is the vector $\boldsymbol{x}_{n}$ cycle shifted by $n$ sampling interval.

By summing up all the vectors $\boldsymbol{x}_{n}^{(n)}, n=0,1, \ldots, N-1$, we can achieve a new signal vector $\boldsymbol{x}_{c s}$, which is defined as

$$
\begin{aligned}
\boldsymbol{x}_{c s}=\sum_{i=0}^{N-1} \boldsymbol{x}_{i}^{(i)}= & {[N x(N-1),(N-1) x(N)+x(0), \cdots,} \\
& \cdots, x(2 N-2)+(N-1) x(N-2)]
\end{aligned}
$$

The new signal vector $\boldsymbol{x}_{c s}$ is then transformed into the frequency domain by DFT.

$$
X_{c s}(m)=\sum_{i=0}^{N-1} \sum_{n=N-1}^{2 N-2} x(n-i) e^{j 2 \pi m i / N} e^{-j 2 \pi m n / N}, m=0,1, . ., N-1
$$

\section{B. Spectrum analysis of discrete exponential signal.}

The discrete exponential signal consisting of $K$ independent modes can be expressed as

$s(n)=\sum_{k=1}^{K} A_{k} e^{-\alpha_{k} n / N} \cos \left(2 \pi f_{k} n / N+\phi_{k}\right), n=0,1, . ., N-1$ where $A_{k}$ is the amplitude, $\phi_{k}$ is the phase, $\alpha_{k}$ and $f_{k}$ are the damping and the frequency, respectively.

Consider the spectra of the original discrete exponential signal $s(n)$ and the pretreated exponential signal $s_{c s}(n)$, respectively.

$$
\begin{array}{r}
S(m)=\sum_{i=0}^{N-1} \sum_{k=1}^{K}\left[A_{k} e^{-\alpha_{k} n / N} \cos \left(2 \pi f_{k} n / N+\phi_{k}\right) e^{-j 2 \pi m n / N}\right] \\
m=0,1, . ., N-1
\end{array}
$$

$$
\begin{aligned}
S_{c s}(m)= & \sum_{i=0}^{N-1} \sum_{n=N-1}^{2 N-2} \sum_{k=1}^{K}\left\{A_{k} e^{-\alpha_{k}(n-i) / N} \times\right. \\
& \left.\cos \left[2 \pi f_{k}(n-i) / N+\phi_{k}\right] e^{j 2 \pi m i / N} e^{-j 2 \pi m n / N}\right\} \\
& m=0,1, . ., N-1
\end{aligned}
$$

In the frequency domain, every mode can be separated into two parts. One part is located at the positive frequencies, the other part is located at the negative frequencies. Therefore, the influence of the signal $s(n)$ and $s_{c s}(n)$ on spectral lines can be expressed as follows:

1)

$$
\begin{array}{r}
S(m)=\sum_{k=0}^{K-1}\left[A_{k}^{+}(m) \angle \phi_{k}^{+}(m)+A_{k}^{-}(m) \angle \phi_{k}^{-}(m)\right], \\
m=0,1, . ., N-1
\end{array}
$$

where [9]

$$
\begin{aligned}
A_{k}^{+}(m) & =\frac{A_{k}}{2} e^{\alpha_{k} \frac{1-N}{2 N}}\left(\frac{\cosh \left(\alpha_{k}\right)-\cos 2 \pi\left(f_{k}-m\right)}{\cosh \left(\alpha_{k} / N\right)-\cos \left(2 \pi\left(f_{k}-m\right) / N\right)}\right)^{\frac{1}{2}} \\
\phi_{k}^{+}(m) & =\phi_{k}-\tan ^{-1} \frac{\sin 2 \pi\left(f_{k}-m\right)}{e^{\alpha_{k}}-\cos 2 \pi\left(f_{k}-m\right)} \\
& +\tan ^{-1} \frac{\sin 2 \pi\left(f_{k}-m\right) / N}{e^{\alpha_{k} / N}-\cos 2 \pi\left(f_{k}-m\right) / N}
\end{aligned}
$$

$$
A_{k}^{-}(m)=\frac{A_{k}}{2} e^{\alpha_{k} \frac{1-N}{2 N}}\left(\frac{\cosh \left(\alpha_{k}\right)-\cos 2 \pi\left(f_{k}+m\right)}{\cosh \left(\alpha_{k} / N\right)-\cos \left(2 \pi\left(f_{k}+m\right) / N\right)}\right)^{\frac{1}{2}}
$$$$
\phi_{k}^{-}(m)=-\phi_{k}+\tan ^{-1} \frac{\sin 2 \pi\left(f_{k}+m\right)}{e^{\alpha_{k}}-\cos 2 \pi\left(f_{k}+m\right)}
$$

$$
-\tan ^{-1} \frac{\sin 2 \pi\left(f_{k}+m\right) / N}{e^{\alpha_{k} / N}-\cos 2 \pi\left(f_{k}+m\right) / N}
$$

$$
\begin{gathered}
S_{c s}(m)=\sum_{k=0}^{K-1}\left[A_{c s k}^{+}(m) \angle \phi_{c s k}^{+}(m)+A_{c s k}^{-}(m) \angle \phi_{c s k}^{-}(m)\right], \\
m=0,1, . ., N-1
\end{gathered}
$$

where (see Appendix A)

$A_{c s k}^{+}(m)=\frac{A_{k}}{2} e^{\alpha_{k} \frac{1-N}{N}}\left(\frac{\cosh \left(\alpha_{k}\right)-\cos 2 \pi\left(f_{k}-m\right)}{\cosh \left(\alpha_{k} / N\right)-\cos \left(2 \pi\left(f_{k}-m\right) / N\right)}\right)$ 


$$
\begin{aligned}
& \phi_{c s k}^{+}(m)= \phi_{k}+\frac{2 \pi\left(f_{k}-m\right)(N-1)}{N}+ \\
& \tan ^{-1} \frac{\sin \left(2 \pi\left(f_{k}-m\right)\right)\left(e^{\alpha_{k}}-e^{-\alpha_{k}}\right)}{2-\cos \left(2 \pi\left(f_{k}-m\right)\right)\left(e^{\alpha_{k}}+e^{-\alpha_{k}}\right)}- \\
& A_{c s k}^{-}(m)=\frac{\tan ^{-1} \frac{\sin \left(2 \pi\left(f_{k}-m\right) / N\right)\left(e^{\alpha_{k} / N}-e^{-\alpha_{k} / N}\right)}{2-\cos \left(2 \pi\left(f_{k}-m\right) / N\right)\left(e^{\alpha_{k} / N}+e^{-\alpha_{k} / N}\right)}}{2} e^{\alpha_{k} \frac{1-N}{N}\left(\frac{\cosh \left(\alpha_{k}\right)-\cos 2 \pi\left(f_{k}+m\right)}{\cosh \left(\alpha_{k} / N\right)-\cos \left(2 \pi\left(f_{k}+m\right) / N\right)}\right)} \\
& \phi_{c s k}^{-}(m)=-\phi_{k}-\frac{2 \pi\left(f_{k}+m\right)(N-1)}{N}- \\
& \tan ^{-1} \frac{\sin \left(2 \pi\left(f_{k}+m\right)\right)\left(e^{\alpha_{k}}-e^{-\alpha_{k}}\right)}{2-\cos \left(2 \pi\left(f_{k}+m\right)\right)\left(e^{\alpha_{k}}+e^{-\alpha_{k}}\right)}+ \\
& \tan ^{-1} \frac{\sin \left(2 \pi\left(f_{k}+m\right) / N\right)\left(e^{\alpha_{k} / N}-e^{-\alpha_{k} / N}\right)}{2-\cos \left(2 \pi\left(f_{k}+m\right) / N\right)\left(e^{\alpha_{k} / N}+e^{-\alpha_{k} / N}\right)}
\end{aligned}
$$

In (9) and (12), the first item on the right-hand side is the influence of modes in the positive frequency, and the second one is the influence of modes in the negative frequency domain. For the spectra of the original discrete exponential signal $s(n)$, the amplitude of the parts in the positive frequency and in the negative frequency are $A_{k}^{+}(m)$ and $A_{k}^{-}(m)$, respectively, and the phase of the parts in the positive frequency and in the negative frequency are $\phi_{k}^{+}(m)$ and $\phi_{k}^{-}(m)$, respectively. For the spectra of the pretreated exponential signal $s_{c s}(n)$, the influence of the parts in the positive frequency on the amplitude and phase spectra are $A_{c s k}^{+}(m)$ and $\phi_{c s k}^{+}(m)$, respectively, and the influence of the parts in the negative frequency on the amplitude and phase spectra are $A_{c s k}^{-}(m)$ and $\phi_{c s k}^{-}(m)$, respectively.

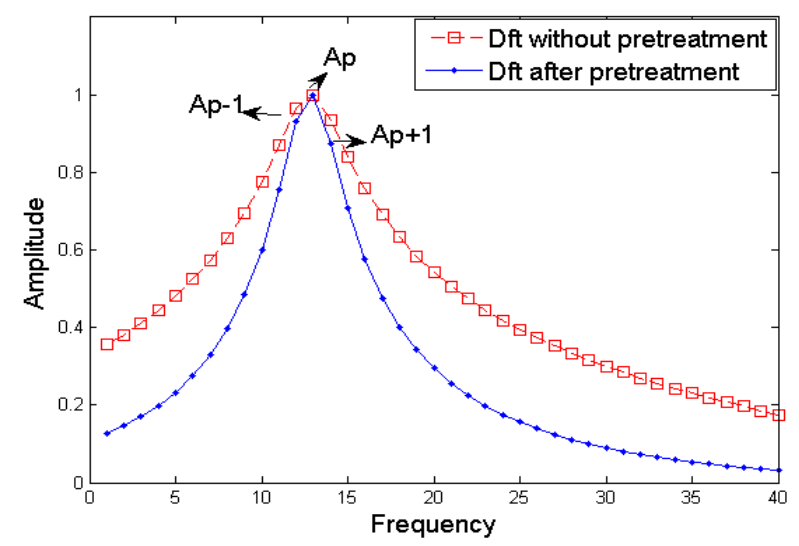

Fig.1. The change of amplitude after pretreatment.
Compare (13) and (10), the term of $A_{c s k}^{+}(m)$ in the brackets is just the square of the term of $A_{k}^{+}(m)$ in the brackets. It is apparent that the amplitude decay near the signal is squared by incorporating the pretreatment in the proposed algorithm, as shown in Fig.1. Therefore, the signal spectrum leakage is effectively reduced.

\section{Parameter estimation.}

In practice, the frequency of the signal lies between the DFT bins with indices: $p-1, p$, and $p+1$, where the DFT bin with index $p$ has the highest magnitude. The energy centralizes near to the spectral line $p$, and its amplitude is particularly obvious. Consequently, the parameters can be calculated on the basis of the three spectral lines on the peak of a mode.

The three amplitudes in a mode are denoted by $A_{p-1}, A_{p}$ and $A_{p+1}$, respectively. Similarly, the reference phase can be obtained from $p$ and donated as $\phi_{p}$. Based on these reference data, the parameters of a mode can be calculated as follows (see Appendix B):

1) Frequency:

$$
\begin{gathered}
f=p+0.5 \frac{A_{p+1}^{-1}-A_{p-1}^{-1}}{2 A_{p}^{-1}-A_{p+1}^{-1}-A_{p-1}^{-1}} \\
\delta=f-p
\end{gathered}
$$

where $\delta$ is the fractional frequency offset.

2) Damping:

$$
\alpha=2 \pi\left(\frac{A_{p+1}(\delta-1)^{2}-A_{p-1}(\delta+1)^{2}}{A_{p+1}-A_{p-1}}\right)^{1 / 2}
$$

3) Amplitude:

$$
A=\frac{A_{p}}{N^{2}} e^{\alpha} \frac{\alpha^{2}+(2 \pi \delta)^{2}}{\cosh (\alpha)-\cos (2 \pi \delta)}
$$

4) Phase:

$$
\begin{gathered}
\phi=\phi_{p}+\tan ^{-1} \frac{\sin (2 \pi \delta / N)\left(e^{\alpha_{k} / N}-e^{-\alpha_{k} / N}\right)}{2-\cos (2 \pi \delta / N)\left(e^{\alpha_{k} / N}+e^{-\alpha_{k} / N}\right)} \\
-\tan ^{-1} \frac{\sin (2 \pi \delta)\left(e^{\alpha_{k}}-e^{-\alpha_{k}}\right)}{2-\cos (2 \pi \delta)\left(e^{\alpha_{k}}+e^{-\alpha_{k}}\right)}-2 \pi \delta
\end{gathered}
$$

\section{SIMULATION AND DISCUSSION}

Here, the performance of the proposed algorithm is validated by simulation and discussed in detail. Firstly, the estimation accuracy of the proposed algorithms is simulated and compared with several algorithms. 
These algorithms for performance comparison, which use adjacent peaks to analyze signal parameters, include the Bertocco algorithm [6], the interpolated discrete Fourier transform for decay (IpDFTd)[11], the interpolated discrete Fourier transform of an exponent (IDFToE) [9], and Yoshida algorithm [8]. They are briefly described as follows:

1) Bertocco algorithm is a frequency-domain interpolation algorithm. The algorithm makes use of two spectral samples.

2) IpDFTd is based on three maximum DFT bin interpolation with the Hanning window.

3) The IDFToE includes two major steps: interpolated DFT and leakage elimination, the first of which gets approximate results. Then, following a few iterations of the second, accurate parameters will be obtained.

4) Taking into account more than three DFT bins, Yoshida algorithm uses four successive DFT bins having the greatest magnitudes.

\section{A. Accuracy.}

To validate the accuracy of the proposed algorithm, the signal of a single-degree-of-freedom dynamic system is first taken as an example to simulate

$$
\begin{array}{r}
s(n)=10 e^{-0.9 n / N} \cos (2 \pi \cdot 35.8 n / N+\pi / 4) \\
n=0,1, . ., 2 N-2
\end{array}
$$

The number of samples is $N=512$, and the proposed algorithms and the other four algorithms are simulated in MATLAB under the same conditions. The estimation results of the five algorithms are shown in Table1.

In Table1., it is obvious that the estimates of the proposed algorithm, including the frequency, the amplitude and the phase, are both closer to the real values compared to the other four algorithms. The performance of the proposed algorithm in damping estimation is slightly inferior to Yoshida and IpDFTd, but better than the others. The performance loss of the proposed algorithm is mainly caused by the trigonometric function approximation in the estimation process which can be found in Appendix B.

Table 1. Comparison of the accuracy of the different algorithms.

\begin{tabular}{|c|c|c|c|c|}
\hline Algorithm & $\begin{array}{c}\text { Freq. } \\
\text { error } \\
(\mathrm{Hz})\end{array}$ & $\begin{array}{c}\text { Damping } \\
\text { error } \\
(1 / \mathrm{sec})\end{array}$ & $\begin{array}{c}\text { Amplitude } \\
\text { error } \\
(\mathrm{pu})\end{array}$ & $\begin{array}{c}\text { Phase } \\
\text { error } \\
(\mathrm{rad})\end{array}$ \\
\hline IDFToE & -0.034 & 0.014 & 0.071 & -0.0114 \\
\hline Yoshida & 0 & 0 & -0.017 & -0.0014 \\
\hline Bertocco & -0.035 & 0.008 & -0.059 & -0.0024 \\
\hline IpDFTd & 0 & 0 & -0.018 & -0.0014 \\
\hline Proposed & 0 & 0.0006 & 0.013 & 0.0003 \\
\hline
\end{tabular}

\section{B. Leakage elimination.}

For the leakage elimination, the superiority of the proposed algorithm which utilizes the preprocessed data to carry out parameter estimation is carefully investigated. Since the IDFToE algorithm reduces the spectral leakage by means of iterative estimation and subtraction of the negative part of the spectrum, its performance of the leakage elimination is superior to Yoshida's and Bertocco's. Therefore, the performance of the proposed algorithm is validated by simulation and compared with the IDFToE. Take a signal as an example, i.e.

$$
\begin{aligned}
s(n)= & 10 e^{-0.5 n / N} \cos (2 \pi \cdot 30.2 n / N-1.047) \\
& +15 e^{-10 n / N} \cos (2 \pi \cdot 60.8 n / N) \quad n=0,1, . ., N-1
\end{aligned}
$$

The number of data is set at $N=512$. The analysis result is recorded in Table 2 .

Table 2. Calculation results of the two algorithms.

\begin{tabular}{|c|c|c|c|c|c|}
\hline \multirow{3}{*}{ Mode } & Parameter & \multirow{2}{*}{ Real Value } & \multicolumn{2}{|c|}{ IDFToE } & \multirow{2}{*}{ Proposed } \\
\cline { 3 - 6 } & & & initial & $5^{\text {th }}$ iteration & \\
\hline \multirow{4}{*}{$\begin{array}{c}\text { Mode } \\
1\end{array}$} & Frequency (Hz) & 30.20 & 30.17 & 30.2 & 30.2 \\
\cline { 2 - 6 } & Damping (1/sec) & 0.500 & 0.857 & 0.5 & 0.497 \\
\cline { 2 - 6 } & Amplitude (pu) & 10.00 & 11.53 & 10.01 & -1.048 \\
\cline { 2 - 6 } Mode & Phase (rad) & -1.047 & -0.92 & 60.8 & 60.8 \\
\cline { 2 - 6 } 2 & Frequency (Hz) & 60.8 & 60.82 & 1048 & 9.99 \\
\cline { 2 - 6 } & Damping (1/sec) & 10.00 & 10.03 & 15.14 & 0.98 \\
\cline { 2 - 6 } & Amplitude (pu) & 15.00 & 14.96 & 0.002 & 0.006 \\
\hline
\end{tabular}

In Table 2., the last column shows the estimates generated by the proposed algorithm. It can be seen that the IDFToE algorithm can obtain the estimates with the same accuracy only after a few iterations. Then, the relationship between the estimation error and the frequency difference of the two modes of the signal is investigated. The estimation error is validated by the Root Mean Square Error (RMSE), which can be calculated as follows:

$$
R M S E=\left\{\frac{1}{E} \sum_{n-0}^{N-1}[\hat{s}(n)-s(n)]^{2}\right\}^{1 / 2}
$$

where

$$
E=\sum_{n-0}^{N-1}[s(n)]^{2}
$$

Estimator $\hat{s}(n)$ is with respect to an estimated signal $s(n)$. 
Assume that both modes have the same damping $\alpha=10$, the same frequency bias $\delta=0.5$ and the same amplitude. When the frequency difference is larger, estimation accuracy is improved. The result of proposed algorithm will reach $-77 \mathrm{~dB}$, while IDFToE algorithm converges to $-47 \mathrm{~dB}$.

Table 3. RMSE of different frequency intervals.

\begin{tabular}{|c|c|c|}
\hline $\begin{array}{c}\text { Frequency } \\
\text { difference }\end{array}$ & $\begin{array}{c}\text { RMSE deviation } \\
\text { of Proposed } \\
(\mathrm{dB})\end{array}$ & $\begin{array}{c}\text { RMSE } \\
\text { deviation of } \\
\text { IDFToE (dB) }\end{array}$ \\
\hline 6 spectral lines & -33.5 & -14 \\
\hline 9 spectral lines & -44 & -44 \\
\hline 12 spectral lines & -52 & -46 \\
\hline 20 spectral lines & -66 & -47 \\
\hline 30 spectral lines & -72.5 & -47 \\
\hline 46 spectral lines & -76.5 & -47 \\
\hline 66 spectral lines & -77 & -47 \\
\hline
\end{tabular}

C. Noise.

In practice, since the measurements are always distorted by wideband noise, the immunity to noise of the considered algorithms is evaluated. The signal with random uniformly distributed frequency and phase which is corrupted by noise is generated. Take a signal as an example, i.e.

$$
\begin{gathered}
s(n)=15 e^{-10 n / N} \cos (2 \pi \cdot 10.8 n / N+\pi / 4) \\
n=0,1, . ., 2 N-2
\end{gathered}
$$

For the given frequency and damping, the test signal was embedded in Gaussian noise with standard deviation corresponding to the assumed signal-to-noise ratio (SNR) from 10 to $50 \mathrm{~dB}$. The RMSE of the estimates generated by the five different methods discussed above are depicted from Fig.2. to Fig.5., respectively.

$$
R M S E_{\text {para }}=\left\{\frac{1}{E} \sum_{n-0}^{N-1}[\hat{\tau}-\tau]^{2}\right\}^{1 / 2}
$$

where

$$
E=\tau^{2}
$$

Estimator $\hat{\tau}$ is with respect to an estimated parameter $\tau$. $\tau$ could be frequency, amplitude, damping or phase.

The RMSE of the frequency and damping estimation results under different SNR are shown in Fig.2. and Fig.3. It can be seen that the proposed algorithm achieves the same accuracy as Yoshida's and IpDFTd's when $S N R \geq 30 \mathrm{~dB}$. Meanwhile, when $S N R \geq 30 d B$, the RMSE of amplitude estimates generated by the proposed algorithm is the smallest of the five algorithms, as shown in Fig.3, and the proposed algorithm has the best amplitude estimation performance. For the phase estimation, the RMSE of the Yoshida and IpDFTd are slightly lower than our method's. This inferiority is mainly caused by the trigonometric approximation incorporated in phase estimation. Thus, the new algorithm has a considerable balanced performance for the noise immunity.

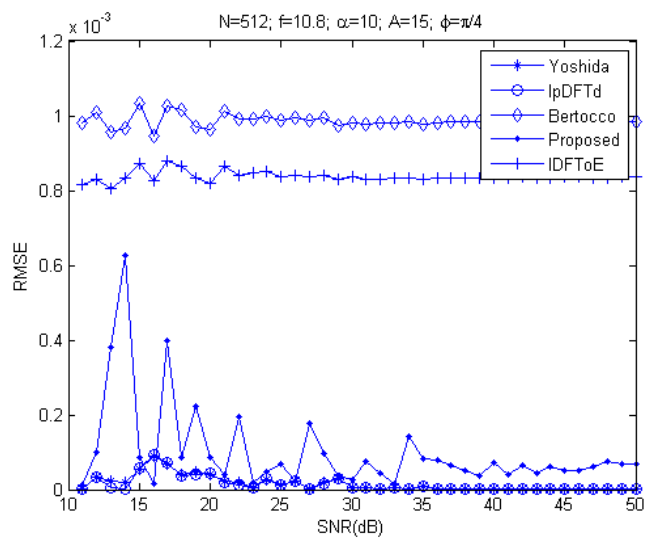

Fig.2. RMSE of frequency estimation under different SNRs.

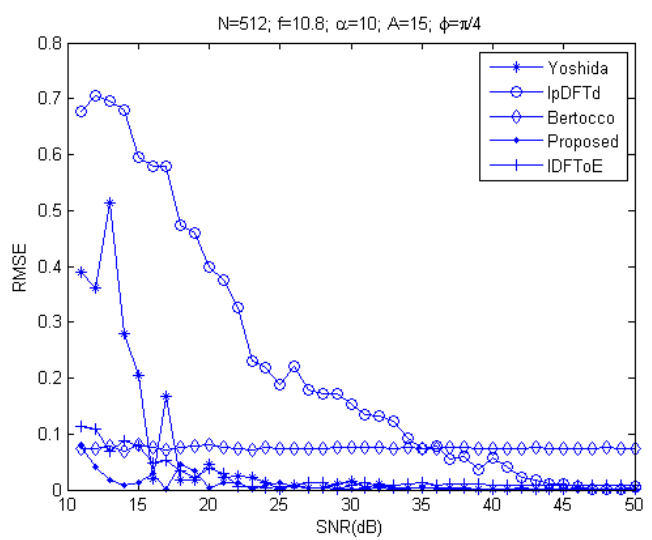

Fig.3. RMSE of damping estimation under different SNRs.

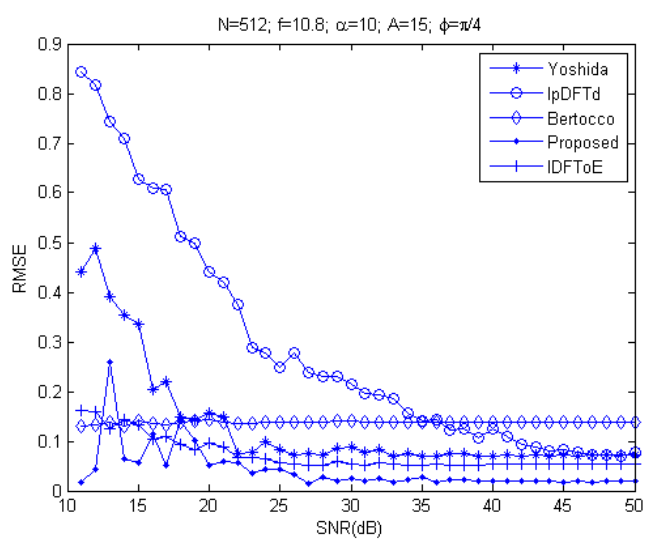

Fig.4. RMSE of amplitude estimation under different SNRs.

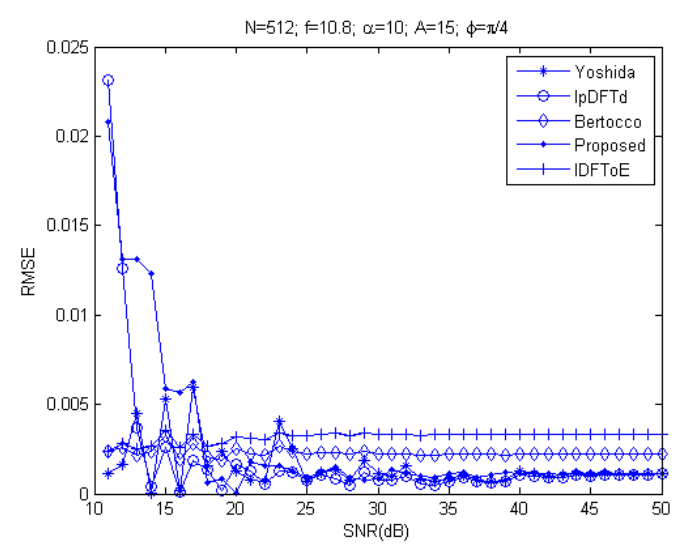

Fig.5. RMSE of phase estimation under different SNRs. 


\section{System error.}

The accuracy and the noise influence on the single parameter estimation have been investigated. The exemplary signal defined as (26) in section 3.C is also utilized here to demonstrate the system error of the discussed algorithms.

Considering all parameters, the systematic error can be calculated using (24) and (25) and is shown in Fig.6.

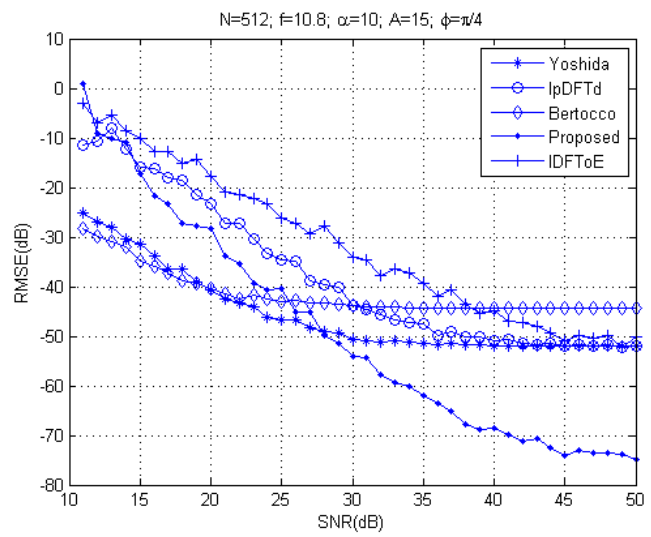

Fig.6. RMSE of signal parameter estimation under different SNRs.

It is very obvious that the performance of the proposed algorithm is significantly improved with the SNR increasing, when $S N R \geq 28 d B$. For a classic condition discussed by the algorithm in [10]: $S N R=38 d B$, the RMSE of the system error analyzed by the proposed algorithm is about $20 \mathrm{~dB}$ lower than the other four algorithms.

Uniform frequency in Fig.7. was changed from 0.04 to 0.16 with the step 0.02 . In Fig.8. damping was changed from 0.004 to 0.01 with the step 0.001 . For each frequency and damping, 150 realizations were generated. The systematic error of the five algorithms is calculated and shown in Figs.7.-8. The RMSE of the systematic error generated by the proposed algorithm is apparently lower than the other four algorithms

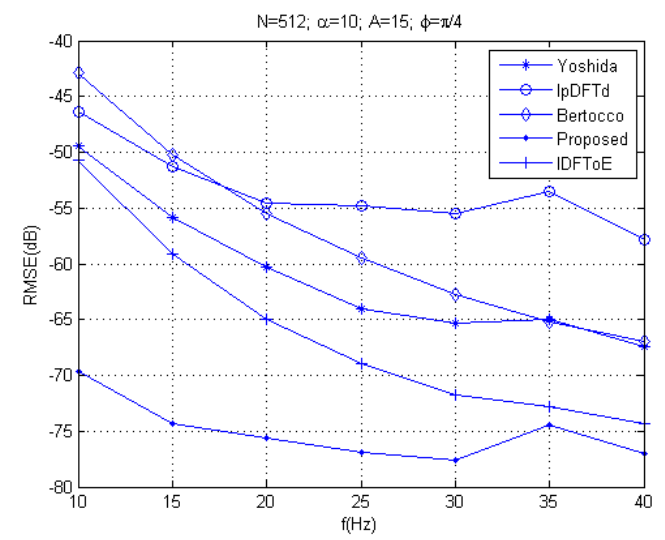

Fig.7. RMSE of signal parameter estimation for different frequencies.

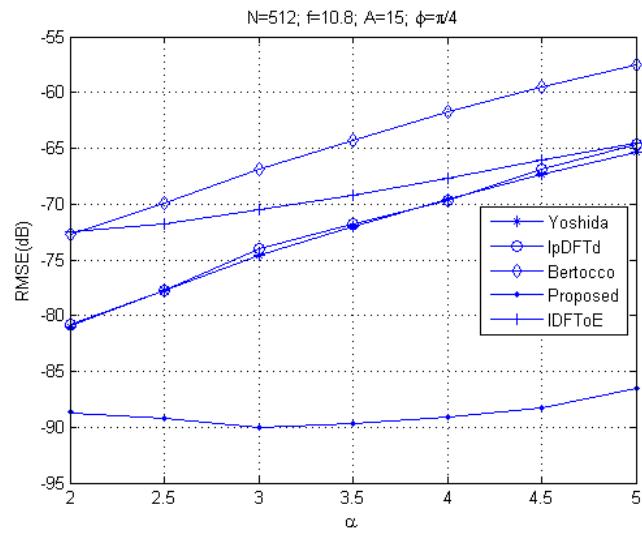

Fig.8. RMSE of signal parameter estimation for damping factors.

Then, the phase $\phi$ is a random variable with uniform distribution in the interval from $-\pi$ to $\pi$. The standard deviations (std) of the estimation errors of frequency and damping are investigated and compared to the Cramér-Rao lower bounds (CRLB). The simulation results are given in Fig.9. and Fig.10., respectively. It can be found that the standard deviations of the estimation errors generated by the proposed algorithm are the lowest among the five algorithms, and closer to CRLB than the other four algorithms.

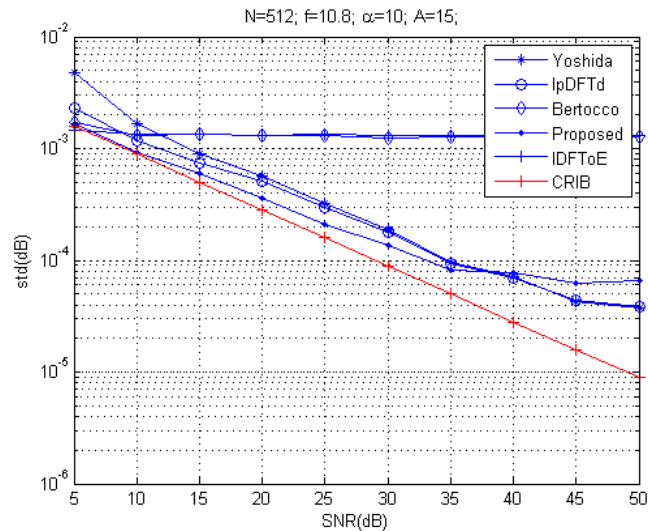

Fig.9. The standard deviation of frequency estimation.

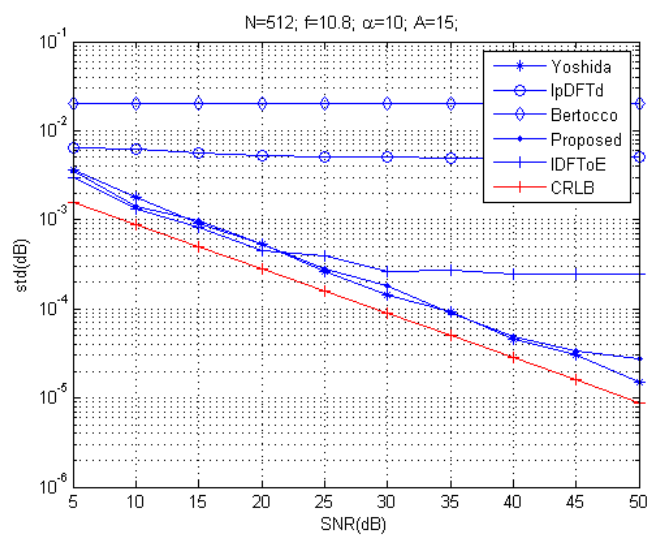

Fig.10. The standard deviation of damping estimation. 


\section{Conclusion}

A novel parameter estimation algorithm for the exponential signal is proposed. The two-step solution first constructs a new signal sequence by continuously cycle shifting and summing up $\mathrm{N}$ buffered exponential signal sample sequences in a preprocessing unit. Then, the interpolation DFT engine is utilized to obtain accurate parameter estimation based on the preprocessed signal sequence. The cycle shift and addition can be quickly and without effort implemented by the processor that has pipeline architecture. New algorithm is computationally efficient. Simulation results indicate that the proposed algorithm employing signal preprocessing can significantly reduce the estimation error in comparison with the existing works, and its performance is hardly affected by spectral leakage effects.

\section{APPENDIX}

\section{A. Spectrum of the preprocessed exponential signal.}

The spectrum of the complex exponential signal can be expressed as

$$
\begin{aligned}
S_{c s}(m)= & \sum_{i=0}^{N-1} \sum_{n=N-1}^{2 N-2} \sum_{k=1}^{K}\left\{A_{k} e^{-\alpha_{k}(n-i) / N} \times\right. \\
& \left.\cos \left[2 \pi f_{k}(n-i) / N+\phi_{k}\right] e^{j 2 \pi m i / N} e^{-j 2 \pi m n / N}\right\}
\end{aligned}
$$

Based on Euler's formula:

$$
\cos \theta=\left(e^{j \theta}+e^{-j \theta}\right) / 2
$$

Expansion of (A.1) is:

$$
\begin{aligned}
S_{c s}(m)= & \frac{1}{2} \sum_{k=1}^{K} A_{k} e^{j \phi_{k}} \sum_{n=N-1}^{2 N-2} e^{-\alpha_{k} n / N} e^{-j 2 \pi m n / N} e^{j\left(2 \pi f_{k} n / N\right)} \times \\
& \sum_{i=1}^{N-1} e^{\alpha_{k} i / N} e^{j 2 \pi m i / N} e^{-j\left(2 \pi f_{k} i / N\right)}+ \\
& \frac{1}{2} \sum_{k=1}^{K} A_{k} e^{-j \phi_{k}} \sum_{n=N-1}^{2 N-2} e^{-\alpha_{k} n / N} e^{-j 2 \pi m n / N} e^{-j\left(2 \pi f_{k} n / N\right)} \times \\
& \sum_{i=1}^{N-1} e^{\alpha_{k} i / N} e^{j 2 \pi m i / N} e^{-j\left(-2 \pi f_{k} i / N\right)}
\end{aligned}
$$

$S_{c s}(m)$ is the sum of two spectra. $S_{c s}^{+}(m)$ is the one for positive frequencies (the first item in the sum) and $S_{c s}^{-}(m)$ is the one for negative frequencies (the second item in the sum).

The exponential signal on spectral lines can be expressed as:

$$
S_{c s}(m)=S_{c s}^{+}(m)+S_{c s}^{-}(m), \quad m=0,1, . ., N-1
$$

By using the formula:

$$
\sum_{n=N-1}^{2 N-2} e^{s n}=\frac{1-e^{s N}}{1-e^{s}} e^{s(N-1)}
$$

Similarly:

$$
\sum_{i=0}^{N-1} e^{s i}=\frac{1-e^{s N}}{1-e^{s}}
$$

$S_{c s}^{+}(m)$ can be expressed as:

$$
\begin{aligned}
S_{c s}^{+}(m)= & \frac{1}{2} \sum_{k=1}^{K}\left[\frac{1-e^{\left(-\alpha_{k}-j 2 \pi m+j 2 \pi f_{k}\right)}}{1-e^{\left(-\alpha_{k}-j 2 \pi m+j 2 \pi f_{k}\right) / N}} A_{k} e^{j \phi_{k}} \times\right. \\
& \left.e^{\left(-\alpha_{k}-j 2 \pi m+j 2 \pi f_{k}\right)(N-1) / N} \cdot \frac{1-e^{\left(\alpha_{k}+j 2 \pi m-j 2 \pi f_{k}\right)}}{1-e^{\left(\alpha_{k}+j 2 \pi m-j 2 \pi f_{k}\right) / N}}\right]
\end{aligned}
$$

The (A.7) can be simplified as:

$$
\begin{aligned}
S_{c s}^{+}(m)= & \frac{1}{2} \sum_{k=1}^{K}\left[\frac{e^{\alpha_{k}}-e^{-j 2 \pi m+j 2 \pi f_{k}}}{e^{\alpha_{k} / N}-e^{\left(-j 2 \pi m+j 2 \pi f_{k}\right) / N}} \cdot A_{k} e^{j \phi_{k}} \times\right. \\
& \left.e^{\left(-\alpha_{k}-j 2 \pi m+j 2 \pi f_{k}\right)(N-1) / N} \cdot \frac{e^{-\alpha_{k}}-e^{j 2 \pi m-j 2 \pi f_{k}}}{e^{-\alpha_{k} / N}-e^{\left(j 2 \pi m-j 2 \pi f_{k}\right) / N}}\right]
\end{aligned}
$$

According to formulas:

$$
\begin{aligned}
\left|e^{\alpha}-e^{j \theta}\right| & =\sqrt{\left(e^{\alpha}-e^{j \theta}\right)\left(e^{\alpha}-e^{-j \theta}\right)} \\
& =\sqrt{2} e^{\alpha / 2}(\cosh \alpha-\cos \theta)^{1 / 2}
\end{aligned}
$$

$S_{c s}^{+}(m)$ can be expressed in amplitude/phase form as

$$
S_{c s}{ }^{+}(m)=\sum_{k=0}^{K-1} A_{c s k}^{+}(m) \angle \phi_{c s k}^{+}(m)
$$

where

$$
\begin{aligned}
& A_{c s k}^{+}(m)= \frac{A_{k}}{2} e^{\alpha_{k} \frac{1-N}{N}} \times \\
& {\left[\frac{\cosh \left(\alpha_{k}\right)-\cos 2 \pi\left(f_{k}-m\right)}{\cosh \left(\alpha_{k} / N\right)-\cos \left(2 \pi\left(f_{k}-m\right) / N\right)}\right] } \\
& \phi_{c s k}^{+}(m)= \phi_{k}+\frac{2 \pi\left(f_{k}-m\right)(N-1)}{N}+ \\
& \tan ^{-1} \frac{\sin \left(2 \pi\left(f_{k}-m\right)\right)\left(e^{\alpha_{k}}-e^{-\alpha_{k}}\right)}{2-\cos \left(2 \pi\left(f_{k}-m\right)\right)\left(e^{\alpha_{k}}+e^{-\alpha_{k}}\right)}- \\
& \tan ^{-1} \frac{\sin \left(2 \pi\left(f_{k}-m\right) / N\right)\left(e^{\alpha_{k} / N}-e^{-\alpha_{k} / N}\right)}{2-\cos \left(2 \pi\left(f_{k}-m\right) / N\right)\left(e^{\alpha_{k} / N}+e^{-\alpha_{k} / N}\right)}
\end{aligned}
$$


The negative frequency part $S_{c s}^{-}(m)$ can be derived in the same way and expressed as

$$
\begin{aligned}
A_{c s k}^{-}(m)= & \frac{A_{k}}{2} e^{\alpha_{k} \frac{1-N}{N}} \times \\
& {\left[\frac{\cosh \left(\alpha_{k}\right)-\cos 2 \pi\left(f_{k}+m\right)}{\cosh \left(\alpha_{k} / N\right)-\cos \left(2 \pi\left(f_{k}+m\right) / N\right)}\right] } \\
\phi_{c s k}^{-}(m)= & -\phi_{k}-\frac{2 \pi\left(f_{k}+m\right)(N-1)}{N}- \\
& \tan ^{-1} \frac{\sin \left(2 \pi\left(f_{k}+m\right)\right)\left(e^{\alpha_{k}}-e^{-\alpha_{k}}\right)}{2-\cos \left(2 \pi\left(f_{k}+m\right)\right)\left(e^{\alpha_{k}}+e^{-\alpha_{k}}\right)}+ \\
& \tan ^{-1} \frac{\sin \left(2 \pi\left(f_{k}+m\right) / N\right)\left(e^{\alpha_{k} / N}-e^{-\alpha_{k} / N}\right)}{2-\cos \left(2 \pi\left(f_{k}+m\right) / N\right)\left(e^{\alpha_{k} / N}+e^{-\alpha_{k} / N}\right)}
\end{aligned}
$$

\section{B. Parameter estimation.}

Parameters are calculated from spectral lines around the component peaks. The data of the spectral lines $p-1, p$ and $p+1$ are substituted for the spectral line $\mathrm{m}$ of $A_{c s k}^{+}(m)$ in (A.11), where the DFT bin with index $p$ has the highest magnitude.

\section{1) Frequency}

By letting the frequency bias $\delta(-0.5<\delta<0.5)$ be

$$
\delta=f-p
$$

For $m=p$

$$
A_{p}=\frac{1}{2} A_{k} e^{\alpha_{k} \frac{1-N}{N}}\left[\frac{\cosh \left(\alpha_{k}\right)-\cos 2 \pi \delta}{\cosh \left(\alpha_{k} / N\right)-\cos (2 \pi \delta / N)}\right]
$$

For $m=p+1$

$$
A_{p+1}=\frac{1}{2} A_{k} e^{\alpha_{k} \frac{1-N}{N}}\left[\frac{\cosh \left(\alpha_{k}\right)-\cos 2 \pi \delta}{\cosh \left(\alpha_{k} / N\right)-\cos (2 \pi(\delta-1) / N)}\right]
$$

For $m=p-1$

$$
A_{p-1}=\frac{1}{2} A_{k} e^{\alpha_{k} \frac{1-N}{N}}\left[\frac{\cosh \left(\alpha_{k}\right)-\cos 2 \pi \delta}{\cosh \left(\alpha_{k} / N\right)-\cos (2 \pi(\delta+1) / N)}\right]
$$

The frequency bias $\delta$ can be calculated based on the amplitude difference.

$$
\begin{aligned}
& A_{p+1}^{-1}-A_{p-1}^{-1}= \frac{2}{A} e^{\alpha \frac{N-1}{N}} \times \\
& \frac{\cos (2 \pi(\delta+1) / N)-\cos (2 \pi(\delta-1) / N)}{\cosh (\alpha)-\cos (2 \pi \delta)} \\
& A_{p}^{-1}-A_{p-1}^{-1}= \frac{2}{A} e^{\alpha \frac{N-1}{N}} \times \\
& \frac{\cos (2 \pi(\delta+1) / N)-\cos (2 \pi \delta / N)}{\cosh (\alpha)-\cos (2 \pi \delta)}
\end{aligned}
$$

Then,

$$
\begin{aligned}
& \frac{A_{p+1}^{-1}-A_{p-1}^{-1}}{2 A_{p}^{-1}-A_{p+1}^{-1}-A_{p-1}^{-1}} \\
= & \frac{\cos (2 \pi(\delta-1) / N)-\cos (2 \pi(\delta+1) / N)}{2 \cos (2 \pi \delta / N)-\cos (2 \pi(\delta-1) / N)-\cos (2 \pi(\delta+1) / N)}
\end{aligned}
$$

By tedious trigonometric function operations, (B.7) can be simplified as:

$$
\frac{A_{p+1}^{-1}-A_{p-1}^{-1}}{2 A_{p}^{-1}-A_{p+1}^{-1}-A_{p-1}^{-1}}=\frac{\tan (2 \pi \delta / N)}{\tan (\pi / N)}
$$

If the variable $\theta$ is sufficiently small, the approximation can be obtained.

$$
\tan \theta \approx \theta
$$

If $N \gg>$ and $N \gg \pi$, the terms $\delta / N$ and $\pi / N$ in (B.8) are all approaching zero. Thus, the frequency bias $\delta$ can be approximated as:

$$
\delta \approx 0.5 \frac{A_{p+1}^{-1}-A_{p-1}^{-1}}{2 A_{p}^{-1}-A_{p+1}^{-1}-A_{p-1}^{-1}}
$$

The frequency can be achieved by substituting (B.10) into (B.1).

$$
\begin{aligned}
f & =p+\delta \\
& =p+0.5 \frac{A_{p+1}^{-1}-A_{p-1}^{-1}}{2 A_{p}^{-1}-A_{p+1}^{-1}-A_{p-1}^{-1}}
\end{aligned}
$$

2) Damping

The damping $\alpha$ can be achieved by (B.3) and (B.4).

$$
\frac{A_{p-1}}{A_{p+1}}=\frac{\cosh (\alpha / N)-\cos (2 \pi(\delta-1) / N)}{\cosh (\alpha / N)-\cos (2 \pi(\delta+1) / N)}
$$


Then, (B.12) can be rewritten as

$$
\begin{aligned}
\cosh (\alpha / N)= & \frac{A_{p-1} \cos (2 \pi(\delta+1) / N)}{A_{p+1}-A_{p-1}}- \\
\frac{A_{p+1} \cos (2 \pi(\delta-1) / N)}{A_{p+1}-A_{p-1}} & -
\end{aligned}
$$

If the variable $\theta$ is sufficiently small, the following approximations can be established.

$$
\begin{aligned}
& \cosh \theta \cong 1+\frac{1}{2} \theta^{2} \\
& \cos \theta \cong 1-\frac{1}{2} \theta^{2}
\end{aligned}
$$

If $N \gg \delta$ and $N \gg \alpha$, the terms $\alpha / N,(\delta-1) / N$ and $(\delta+1) / N$ in (B.13) are all approaching zero. Thus, the (B.13) can be approximated as

$$
(\alpha / N)^{2}=\frac{A_{p+1}[2 \pi(\delta-1) / N]^{2}-A_{p-1}[2 \pi(\delta+1) / N]^{2}}{A_{p+1}-A_{p-1}}
$$

The damping $\alpha$ can be expressed as

$$
\alpha=2 \pi\left[\frac{A_{p+1}(\delta-1)^{2}-A_{p-1}(\delta+1)^{2}}{A_{p+1}-A_{p-1}}\right]^{1 / 2}
$$

\section{3) Amplitude}

The amplitude can be derived from (B.2)

$$
A=2 A_{p} e^{\alpha_{k} \frac{N-1}{N}}\left[\frac{\cosh (\alpha / N)-\cos (2 \pi \delta / N)}{\cosh (\alpha)-\cos (2 \pi \delta)}\right]
$$

If $N>>\delta$ and $N>>\alpha,($ B.18) can be simplified according to (B.14) and (B.15).

$$
A=2 A_{p} e^{\alpha}\left[\frac{0.5(\alpha / N)^{2}+0.5(2 \pi \delta / N)^{2}}{\cosh (\alpha)-\cos (2 \pi \delta)}\right]
$$

The amplitude can be expressed as

$$
A=\frac{A_{p}}{N^{2}} e^{\alpha} \frac{\alpha^{2}+(2 \pi \delta)^{2}}{\cosh (\alpha)-\cos (2 \pi \delta)}
$$

\section{4) Phase}

For $m=p,(\mathrm{~A} .12)$ can be expressed as

$$
\begin{aligned}
\phi_{p}= & \phi-\tan ^{-1} \frac{\sin (2 \pi \delta / N)\left(e^{\alpha_{k} / N}-e^{-\alpha_{k} / N}\right)}{2-\cos (2 \pi \delta / N)\left(e^{\alpha_{k} / N}+e^{-\alpha_{k} / N}\right)} \\
& +\tan ^{-1} \frac{\sin (2 \pi \delta)\left(e^{\alpha_{k}}-e^{-\alpha_{k}}\right)}{2-\cos (2 \pi \delta)\left(e^{\alpha_{k}}+e^{-\alpha_{k}}\right)}+\frac{2 \pi \delta(N-1)}{N}
\end{aligned}
$$

If $N$ is large enough, $\frac{(N-1)}{N} \approx 1$. (B.21) can be simplified as

$$
\begin{gathered}
\phi=\phi_{p}+\tan ^{-1} \frac{\sin (2 \pi \delta / N)\left(e^{\alpha_{k} / N}-e^{-\alpha_{k} / N}\right)}{2-\cos (2 \pi \delta / N)\left(e^{\alpha_{k} / N}+e^{-\alpha_{k} / N}\right)} \\
-\tan ^{-1} \frac{\sin (2 \pi \delta)\left(e^{\alpha_{k}}-e^{-\alpha_{k}}\right)}{2-\cos (2 \pi \delta)\left(e^{\alpha_{k}}+e^{-\alpha_{k}}\right)}-2 \pi \delta
\end{gathered}
$$

\section{ACKNOWLEDGMENT}

This work was supported by the Fundamental Research Funds for the Central Universities, China (grant \# ZYGX2012J146) and the Specialized Research Fund for the Doctoral Program of Higher Education, China (grant \# 20130185110023).

\section{REFERENCES}

[1] Aboutanios, E. (2011). Estimating the parameters of sinusoids and decaying sinusoids in noise. IEEE Instrumentation \& Measurement Magazine, 14 (2), 814.

[2] Aboutanios, E. (2010). Estimation of the frequency and decay factor of a decaying exponential in noise. IEEE Transactions on Signal Processing, 58 (2), 501509.

[3] Duda, K., Zielinski, T.P. (2013). Efficacy of the frequency and damping estimation of a real-value sinusoid Part 44 in a series of tutorials on instrumentation and measurement. IEEE Instrumentation \& Measurement Magazine, 16 (2), 4858.

[4] Agrez, D. (2009). A frequency domain procedure for estimation of the exponentially damped sinusoids. In Instrumentation and Measurement Technology Conference, 5-7 May 2009. IEEE, 1321-1326.

[5] Zieliński, T., Duda, K. (2011). Frequency and damping estimation methods-an overview. Metrology and Measurement Systems, 18 (4), 505-528.

[6] Bertocco, M., Offelli, C., Petri, D. (1994). Analysis of damped sinusoidal signals via a frequency-domain interpolation algorithm. IEEE Transactions on Instrumentation and Measurement, 43 (2), 245-250.

[7] Agrež, D. (2010). Estimation and tracking of the power quality disturbances in the frequency domain. Measurement Science Review, 10 (6), 189-194. 
[8] Yoshida, I., Sugai, T., Tani, S., Motegi, M., Minamida, K., Hayakawa, H. (1981). Automation of internal friction measurement apparatus of inverted torsion pendulum type. Journal of Physics E: Scientific Instruments, 14 (10), 1201.

[9] Wu, R.C., Chiang, C.T. (2010). Analysis of the exponential signal by the interpolated DFT algorithm. IEEE Transactions on Instrumentation and Measurement, 59 (12), 3306-3317.

[10] Agrez, D. (2002). Weighted multipoint interpolated DFT to improve amplitude estimation of multifrequency signal. IEEE Transactions on Instrumentation and Measurement, 51 (2), 287-292.
[11] Duda, K., Magalas, L.B., Majewski, M., Zielinski, T.P. (2011). DFT-based estimation of damped oscillation parameters in low-frequency mechanical spectroscopy. IEEE Transactions on Instrumentation and Measurement, 60 (11), 3608-3618.

[12] Diao, R., Meng, Q. (2013). An interpolation algorithm for discrete Fourier transforms of weighted damped sinusoidal signals. IEEE Transactions on Instrumentation and Measurement, 99, 1-9.

[13] Duda, K. (2011). DFT interpolation algorithm for Kaiser-Bessel and Dolph-Chebyshev windows. IEEE Transactions on Instrumentation and Measurement, 60 (3), 784-790. 